\title{
Erratum: Diabetic retinopathy and retinal screening awareness amongst female diabetic patients at a day hospital diabetic clinic in Cape Town, South Africa
}

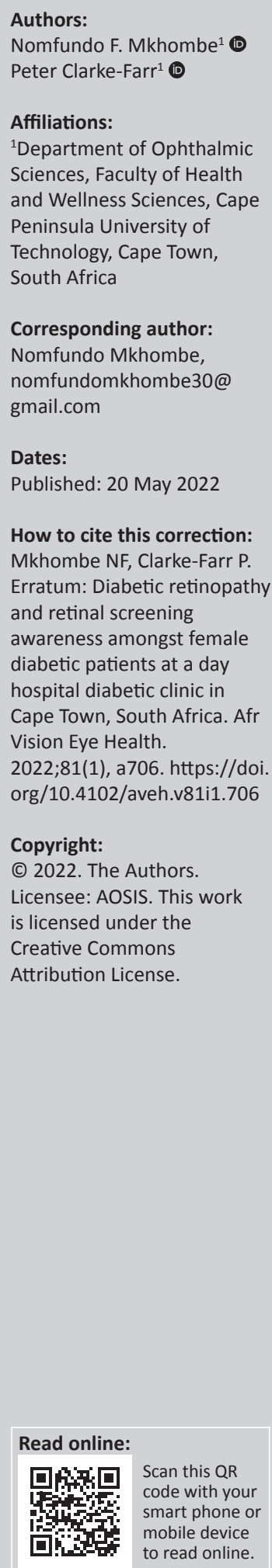

Corresponding author: Nomfundo Mkhombe, nomfundomkhombe30@ gmail.com

Dates:

Published: 20 May 2022

How to cite this correction: Mkhombe NF, Clarke-Farr P. Erratum: Diabetic retinopathy and retinal screening awareness amongst female diabetic patients at a day hospital diabetic clinic in Cape Town, South Africa. Afr Vision Eye Health. 2022;81(1), a706. https://doi. org/10.4102/aveh.v81i1.706

\section{Copyright:}

(C) 2022. The Authors.

Licensee: AOSIS. This work

is licensed under the

Creative Commons

Attribution License.

In the published article, Mkhombe NF, Clarke-Farr P. Diabetic retinopathy and retinal screening awareness amongst female diabetic patients at a day hospital diabetic clinic in Cape Town, South Africa. Afr Vision Eye Health. 2021;80(1), a614. https://doi.org/10.4102/aveh.v80i1.614, there was an error in the affiliation for both authors. Instead of 'Department of Ophthalmic Sciences, Faculty of Health and Awareness Sciences, Cape Peninsula University of Technology, Cape Town, South Africa', it should be 'Department of Ophthalmic Sciences, Faculty of Health and Wellness Sciences, Cape Peninsula University of Technology, Cape Town, South Africa'.

The publisher apologises for this error. The correction does not change the study's findings of significance or overall interpretation of the study's results or the scientific conclusions of the article in any way. 


\section{Diabetic retinopathy and retinal screening awareness amongst female diabetic patients at a day hospital diabetic clinic in Cape Town, South Africa}

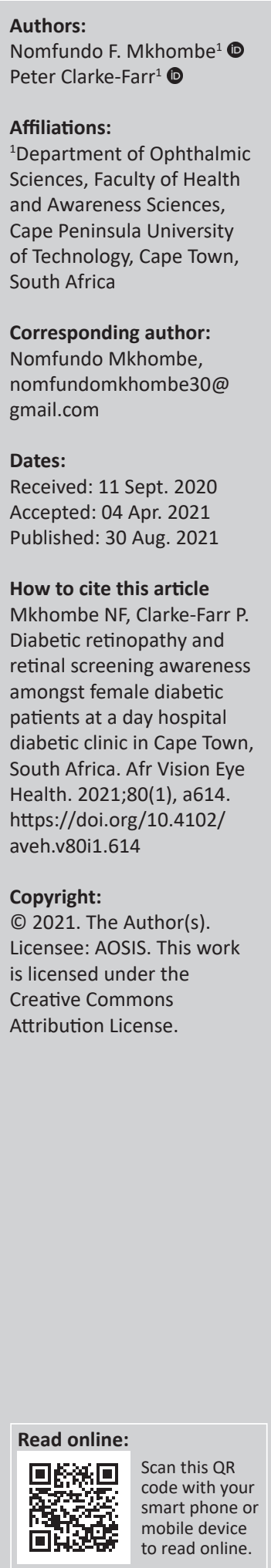

Background: Most eye complications presenting at a diabetic clinic are preventable; however, few patients are aware of the importance of regular diabetic eye examinations. Diabetes causes diabetic retinopathy (DR), yet retinal screening provides an opportunity to refer for appropriate treatment and prevention of avoidable blindness.

Aim: The aim of this research was to evaluate the awareness levels of DR and retinal screening procedures amongst female diabetic patients.

Setting: The study was conducted at Site B day clinic, situated in Khayelitsha in Cape Town, South Africa.

Methods: Data collection involved a self-developed and administered questionnaire. Two research assistants, trained in ethical research, assisted in administering the instrument and data collection over an 8-week period.

Results: Data were collected from 149 randomly selected female diabetic patients. Approximately, three-quarters $(71.1 \%, n=106)$ had knowledge about DR and the importance of retinal screening, and most $(79.2 \%, n=118)$ agreed that diabetic disease can lead to blindness. Whilst $63.1 \%(n=94)$ of respondents understood the importance of screening, just over three-quarters $(75.8 \%, n=113)$ felt that all people should attend retinal screening even though $80.4 \%(n=119)$ agreed that DR leads to permanent eye damage. Significantly, $60.4 \%$ $(n=90)$ felt that attending retinal screening sessions may delay the need for spectacles. Just over half $(53.0 \%, n=79)$ felt that cultural and spiritual beliefs influenced the respondents' attendance for retinal screening.

Conclusion: Whilst respondents were, in general, well aware of the risks associated with diabetes mellitus (DM) and DR and knowledge of retinal screening was relatively good, the survey found that in the respondents' opinion, attending the retinal screening caused delays in getting new spectacles because this screening was a prerequisite for referral for spectacles.

Keywords: awareness; diabetes mellitus; diabetic retinopathy; female patients; retinal screening.

\section{Introduction}

Globally, an estimated 422 million adults were living with diabetes in 2014, compared with 108 million in $1980^{1}$ and this increase was largely associated with increasing obesity. According to Harding et al. ${ }^{2}$ the increase in the number of people with diabetes and the longer duration of diabetes is likely to alter the disease profile in many populations around the globe, which consequently impacts on the quality of life, demands on health services and costs to the economy. ${ }^{2}$ The ocular changes that occur in diabetic patients affect nearly the entire eye, and the most significant ocular sequelae caused by diabetes mellitus (DM) are refractive changes, cataracts, rubeosis iridis, ocular hypertension and diabetic retinopathy (DR). ${ }^{3}$ Furthermore, secondary glaucoma, colour vision changes and neuropathies are all consequences of diabetic disease. ${ }^{4}$ Microvascular complications of the retina, which later manifest as DR, constitute the fifth leading cause of blindness and are responsible for $4.8 \%$ of the total causes of blindness worldwide, affecting approximately 1.8 billion people..$^{5}$ Of critical concern is that this prevalence of DR is increasing at an alarming rate worldwide. ${ }^{6}$

As a result of the time lapse between the onset of diabetes and the diagnosis thereof, patients with Type 2 DM may have significant DR at first diagnosis, compared with patients with Type 1 DM. ${ }^{7}$ 
The pathogenesis of retinopathy in DM is still not fully understood, but hypertension, poor glycaemic control and increasing duration of DM have been identified as risk factors for DR. ${ }^{7}$ Diabetic retinopathy is classified according to its severity, which progresses from mild non-proliferative to proliferative DR (PDR); it is the most serious form, which leads to blindness. At various stages of DR, macula oedema, which is characterised by swelling of the retina, occurs because of the leakage of blood vessels of the retina. ${ }^{8}$ Successful treatment programmes in communities depend largely on the awareness of that disease by the general community, as established by a study conducted in a Kenyan Hospital. ${ }^{9}$ There, it was found that $83 \%$ of the participants had heard of DR, and $60 \%$ of those who knew of diabetic eye disease were aware of the relation between diabetic eye disease and diabetes. However, the researchers found that there was little knowledge about the risk and prevention strategies for the development of DR.

The process of retinal screening is described as not only allowing for the detection of microvascular complications, but for the detection of other comorbidities as well. Diabetic retinal screenings are defined as an annual eye check-up to identify early signs of DR. ${ }^{10}$ Previously, direct fundoscopy was the method of examining the fundus at primary healthcare level and although few healthcare workers are equipped with this skill there seems a major need for this procedure for diabetic patients. ${ }^{11}$ There is evidence that few patients are being screened for DR, which results in a decreased awareness of diabetic eye complications and the importance of annual retinal screening. There is also a high default rate among those patients who were screened, thus reflecting a poor understanding of the necessity for retinal screening and ignorance of the condition.

In Malaysia, one study assessed the awareness of eye complications and the prevalence of DR amongst Type 2 diabetic patients attending a tertiary medical centre in Kuala Lumpur. It was found that $86 \%$ of the respondents were aware of diabetic eye complications, especially patients who had achieved tertiary educational level. ${ }^{12}$ However, $43.8 \%$ of the participants did not know how frequently they should go for an eye test and $72.3 \%$ did not know what treatments were available. A lack of understanding of the diabetic diseases was a barrier for most diabetic patients, and this led to delays in their eye tests and retinal screening. ${ }^{12}$ In Africa, findings from a Nigerian study ${ }^{13}$ revealed that DR had a prevalence of $33.0 \%$ in the accessible population, which accounted for $16.7 \%$ of retinal disease, and had led to bilateral blindness in $22.0 \%$ of diabetic patients. This prevalence depended on both the duration of diabetes and the level of glycaemic control. Findings from their study also showed that a diabetic patient who had strict and well-controlled blood sugar delayed the onset of developing DR and retarded the progression of established diabetic complications in the entire body. ${ }^{13}$

In South Africa, DR is the third leading cause of blindness, preceded only by cataract and glaucoma and is responsible for $5 \%$ of all cases of blindness. It was found that Cape Town as a case in time was falling well short of the recommended retinal screening protocols for diabetics and that there was a general lack of awareness amongst diabetic patients concerning the existence of diabetic eye complication and the importance of retinal screening. ${ }^{5}$ South Africa has lagged behind other countries because of the increased pressures of other primary healthcare demands, resulting in increasing numbers of diabetics not having sufficient opportunities to be screened. More recently, however, the Ophthalmological Society of South Africa has developed a low-cost system for national retinal screenings. ${ }^{11}$ The South African public sector tends to follow a structural model with particular pitfalls; for example, there are no referrals, no structured central register, no recall system or a common grading system within the public sector. Inversely, the private sector follows an opportunistic model, which entails an annual structured retinal-screening recall system by an optometrist, with record keeping and further analysis and possible referrals to an ophthalmologist. This latter model is a far better approach because there is direct, swift communication between the optometrist and the ophthalmologist regarding a specific patient.

The research on which this article has drawn was based on the determinants of the Health Belief Model (HBM), ${ }^{14}$ a model that provides a framework for understanding why some people take particular actions to avoid illness and inversely why others fail to protect themselves. This model is beneficial in the assessment of health protection or disease prevention behaviours, the organising of information regarding clients' views of their state of health and the factors that influence them to make a change in their behaviour. The HBM is also appropriately used in providing organised assessment of data regarding the clients' abilities and motivation to change their health status. It includes four constructs that are combined to predict health-related beliefs as applied in the context of the present study. These beliefs comprise individual perceptions, modifying factors, demographic factors and socio-psychological factors. ${ }^{14}$

Different female diabetic patients who know they are diabetic have different perceptions of how to take care of themselves. Some of them find it challenging, which often causes patients to become depressed and enter into panic modes of emotions. This, in turn, often leads to uncontrolled glycaemic levels. Socioeconomic factors may also play a role when female diabetic patients do not have access to a balanced diet. They may, for instance, not be able to afford a balanced meal and may have more carbohydrates in their diet, which increases their glycaemic levels. Cultural factors such as female responsibilities in society may also play a role, where most of the elderly females are unemployed or retired and are forced to remain at home and look after their grandchildren. These elderly women tend to attend appointments poorly or sporadically and often fail to appear for retinal screening referrals or collection of their medication from the day hospital. As these elderly women are virtually on their own 
at home, they forget to take their medication or some of those on insulin are unable to administer it themselves, thus causing an increase in glycaemic levels and consequently the risk of further developing DR. ${ }^{15}$

Although blindness caused by DR is preventable, the experience of the researcher is that a notable number of female patients report at eye clinics when they are already in advanced stages of diabetes with DR. Some of the diabetic female patients do have retinal screening performed on their eyes, but there is no follow-up. When requested to return for follow-ups they do not return perhaps because of insufficient knowledge and awareness of DR and its importance. As a result, patients in the public sector are often not referred for screening. This may be because of the primary caregiver who does not think of doing so and the HBM would help these patients to understand the importance of retinal screening. The research question of the present study was therefore based on the population, intervention, comparison and intervention (PICO) approach and formulated as follows: What is the awareness level of female diabetic patients attending the diabetic clinic in a day hospital about the occurrence of DR and diabetic retinal screening? Based on this, four research objectives guided the study: to evaluate female diabetic patients' knowledge of DM, to evaluate their knowledge pertaining to DR, to elicit their level of awareness pertaining to diabetic retinal screening and to determine their awareness of the risk factors associated with DM and DR.

\section{Methodology}

An observational, descriptive and contextual study design was employed for this research. The data were collected over a period of eight weeks. Khayelitsha is part of an informal settlement situated on the Cape Flats between Table Bay and False Bay in the Western Cape Province, South Africa. The total population of the area is about 391749 people. In and around Khayelitsha there are approximately four day-clinics that provide healthcare services. Khayelitsha Site B, which is also referred to as Nonqubela, was the day clinic utilised to collect data in the present study. ${ }^{16}$ Health services, including annual retinal eye screenings, are provided to all diabetic patients once a week on Mondays. The target population comprised all female diabetic patients who attended the diabetic clinic in Khayelitsha on the days when data collection took place.

The inclusion criteria for the study were female patients between 30 and 60 years of age, who attended the diabetic clinic at the day hospital and who may or may not have undergone diabetic retinal screening (both Type $1 \mathrm{DM}$ and Type 2 DM). Exclusion criteria were that participants should not be younger than 30 or over 60 years of age, not members of the day clinic and not male diabetic patients. The reason for these exclusions was that females predominantly attend retinal screening at the clinic and that this study aimed to consider primarily female knowledge and awareness.
An original structured survey questionnaire was developed for the purpose of data collection. The instrument was based on a comprehensive review of the determinants of health behaviour. A pilot study, pretesting the instrument, was conducted on five patients two weeks before the actual data collection began. The questionnaire consisted of 11 items, comprising the respondents' socio-demographic characteristics, family history of DM, the period during which respondent had been diabetic, general health status, eye operations, wearing of spectacles and satisfaction with eye information provided at the clinic. Two trained volunteer research assistants administered the instrument and assisted the prime investigator in collecting data over a period of eight consecutive weeks, as the diabetic clinic runs at the day hospital once a week on Mondays. The researcher provided all the equipment needed for the study whilst the diabetic club provided a quiet room for questionnaire completion. Data were analysed by using the Statistical Package for the Social Sciences (SPSS) software programme in consultation with a statistician from the University of South Africa (UNISA). Descriptive statistics in terms of frequencies, means and standard deviations were used to analyse the demographic information as well as the direct responses to the questionnaire. Chi-square tests and inferential statistics were used to explore patterns of association in response to the survey questions.

\section{Ethical considerations}

Ethical approval for the research was granted by the Research and Ethics Committee of the Department of Health Studies at the University of South Africa (HRHDC/289/293). This research took place under the authority of the management of the chosen day clinic, and permission was obtained from the facility management. The researcher protected the rights of all the participants in terms of obtaining informed consent, voluntary participation and ensuring participants' selfdetermination, privacy, anonymity and confidentiality, fair treatment and justice and the freedom to withdraw from the study at any given time without experiencing prejudice and/ or victimisation. ${ }^{17}$

\section{Results}

Approximately half of the sample 54.4\% $(n=81)$ were between 51 and 60 years of age at the time of the survey, which was also the median age group. Slightly more than half of the respondents $(56.5 \%, n=84)$ were single, divorced or widowed. As expected, as the research was conducted in Khayelitsha, 91.3\% $(n=136)$ belonged to the isiXhosa language group. Not many of the respondents had had formal education: only $21.5 \%(n=32)$ had completed Grade 11 or 12 , with a low percentage of $24.2 \%(n=36)$ having had some form of tertiary education. Possibly, because of the lower levels of education amongst these respondents, $38.8 \%$ $(n=57)$ were unemployed and only $23.1 \%(n=34)$ reported being employed or self-employed. 
With respect to having a family history of diabetes, 58\% $(n=80)$ reported in the affirmative, whereas $42 \%(n=58)$ reported in the negative. A total of 11 respondents did not answer the question. The reason for this might have been that they did not understand the question or perhaps they preferred not to disclose this information for fear of prejudice. Only $8.2 \%(n=12)$ of the 147 respondents reported that they had had diabetes for less than 1 year. Those who had been diabetic for 1-2 years constituted $17.7 \%(n=26)$, those who had been diabetic for 3-4 years made up $17.7 \%$ $(n=26)$, whilst $21.9 \%(n=31)$ had been diabetic for 5-6 years. In addition, those who had had diabetes for a duration of $7-8$ years totalled $10.9 \%(n=16)$. However, the bulk of the respondents, comprising $24.5 \%(n=36)$, had been diabetic for more than eight years.

Concerning the general health status of the respondents, 9.52\% $(n=14)$ reported excellent health, whilst $32.6 \%$ $(n=48)$ reported their general health as good. Respondents who reported their health as 'average' totalled $28.6 \%$ $(n=42)$, whilst those who reported their general health being 'poor' comprised 8.8\% $(n=13)$. The 32.6\% $(n=48)$ of respondents who reported their general health as 'good' were the ones who were well informed about DM. They also accounted for the respondents who agreed that eating a healthy diet contributes to their quality of life (96\%, $n=143$ ) and that doing regular exercise kept them healthy $(91.4 \%, n=137)$. These respondents also stated that smoking $(77.9 \%, n=116)$ and drinking $(80.5 \%, n=120)$ can elevate one's blood sugar levels. With respect to their ocular health, $18.8 \%(n=28)$ of the respondents had previously undergone some form of eye operations or procedures. The other $76.5 \%(n=114)$ did not have a history of eye operations and procedures.

Regarding the use of spectacles, $15.1 \%(n=22)$ of the respondents reported wearing spectacles only when they were reading. These were the older patients over 40 years of age and who were experiencing presbyopia. The $39.7 \%$ $(n=58)$ who reported wearing spectacles constantly were those who had refractive errors and used their spectacles all the time to correct their vision both at a distance and near. Concerning eye information provided by the clinical staff to patients, $32.2 \%(n=48)$ of the respondents reported being 'very satisfied', whilst $53.0 \%(n=79)$ reported being 'just satisfied'. There were some respondents indicated being undecided about this issue $(10.1 \% ; n=15)$, whilst $4.8 \%(n=7)$ reported being 'dissatisfied' with the eye information provided to them at the diabetic clinic.

The second part of the survey dealt with the respondents' general health and DM knowledge and offered three choices on a three-point Likert scale ranging from 'agree' to 'undecided' to 'disagree'. Table 1 summarises the respondents' general health and their knowledge of DM.

Most respondents $(79.9 \%, n=119)$ agreed that DM was related to high sugar intake, whilst $13.4 \%(n=20)$ disagreed that DM was related to high sugar intake. According to their knowledge of DM and general health, $79.2 \%(n=118)$ of the respondents agreed that DM could cause blindness, and these were the respondents who reported having had DM for more than 8 years $(24.49 \%, n=36)$ and who were also very satisfied with the information that was given to them at the club pertaining to DM. Whilst $9.4 \%(n=14)$ of the respondents disagreed with the statement, these were the respondents who reported having had DM for less than one year $(8.16 \%$, $n=12$ ), had been recently diagnosed and had just begun attending the diabetes club. The knowledge that they had about DM and their eyes was therefore still limited.

For the question related to medication, 63.0\% $(n=94)$ disagreed with the statement that when diabetic patients feel better, they are allowed to stop their medication. This is a good indication of compliance and adherence to treatment. The $24.1 \%(n=36)$ of the respondents who agreed with the statement either did not understand the question, or this might demonstrate a lack of knowledge about DM and its treatment or management. With regard to obesity, almost half of the respondents $(49.3 \%, n=73)$ agreed with the statement that DM is more common in obese people. Similarly, almost half of the respondents $(49.3 \%, n=73)$ reported that they agreed that DM is more common in women compared with men, whilst 31.5\% $(n=47)$ were undecided. The respondents who agreed with the statement that compared DM prevalence in adults with children were $53.7 \%(n=80)$. The last statement in this section pertained to how poor eyesight because of DM affects the quality of life. Almost three-quarters $(71.1 \%, n=106)$ of the respondents agreed with this statement, which indicates that these respondents had a good understanding of the consequences of DR and how it can affect one's quality of life. When

TABLE 1: General health and diabetes mellitus knowledge $(n=149)$.

\begin{tabular}{|c|c|c|c|c|c|c|c|}
\hline \multirow[t]{3}{*}{ Subset of questions that describe general knowledge } & \multicolumn{7}{|c|}{ Agreement levels } \\
\hline & \multicolumn{2}{|c|}{ Agree } & \multicolumn{2}{|c|}{ Undecided } & \multicolumn{2}{|c|}{ Disagree } & \multirow[t]{2}{*}{ Total } \\
\hline & $n$ & $\%$ & $n$ & $\%$ & $n$ & $\%$ & \\
\hline 1.1: DM is related to high sugar intake. & 119 & 79.8 & 10 & 6.7 & 20 & 13.4 & 149 \\
\hline 1.2: DM disease can lead to blindness. & 118 & 79.2 & 10 & 6.7 & 21 & 14.1 & 149 \\
\hline 1.3: When you feel better, you are allowed to stop the DM medication. & 36 & 24.1 & 19 & 12.7 & 94 & 63.0 & 149 \\
\hline 1.4: DM is more common in obese (fat) people. & 73 & 49.3 & 23 & 15.5 & 52 & 35.1 & 148 \\
\hline 1.5: $\mathrm{DM}$ is more common in women than in men. & 72 & 48.3 & 30 & 20.1 & 47 & 31.5 & 149 \\
\hline 1.6: DM mellitus is more common in adults than in children. & 80 & 53.7 & 16 & 10.7 & 53 & 35.6 & 149 \\
\hline 1.7: Poor eyesight because of DM affects the quality of life. & 106 & 71.1 & 15 & 10.1 & 28 & 8.8 & 149 \\
\hline Total & 604 & - & 123 & - & 315 & - & 1042 \\
\hline
\end{tabular}

DM, diabetes mellitus. 
conducting the chi-square test of the response patterns, the value was 179.02. For $d f=24$, the associated $p$-value was well below 0.0001, indicating that the frequency response patterns differed statistically.

The second section of the questionnaire dealt with the participants' knowledge pertaining to DR. Table 2 summarises the data in this respect.

The vast majority, comprising $80.4 \%(n=119)$ of the respondents, agreed with the statement that DR leads to permanent eye damage, whilst only $12.1 \%(n=18)$ were undecided about this statement. The respondents who agreed with the statement indicated that they had knowledge regarding DR. These were also the respondents who had had a formal education, which included those who had completed Grade 11 or 12 and tertiary education $(24.1 \%, n=36)$. Many respondents $(71.6 \%, n=106)$ also agreed that DR was well known in their community. Furthermore, a large number of respondents $(71.8 \%, n=$ 107) also agreed that DR is common in people who have had DM for a long time. An important finding from this section relates to how an awareness of DR seemingly helps people to seek timeous assistance. Here, $78.5 \%(n=117)$ of the respondents agreed with the statement, whilst only $13.4 \%(n=20)$ were undecided and $8 \%(n=12)$ disagreed with the statement.

Just over half the respondents at 53\% $(n=79)$ agreed with the statement on how cultural or spiritual beliefs may be a reason why people do not attend retinal screening, whilst $33.5 \%(n=50)$ of the respondents disagreed with the statement.

The third section of the questionnaire dealt with the participants' knowledge pertaining to DR. Table 3 illustrates the respondents' level of awareness pertaining to diabetic retinal screening.

Overall, almost two-thirds $(63.0 \%, n=94)$ of the respondents agreed that people in their community understood the importance of retinal screening. This kind of response indicates some knowledge about the diabetic clinic and retinal screening. Of the respondents, $85.9 \%(n=$ 128 ) indicated that there was a diabetic clinic within their health facility and $85.2 \%(n=127)$ observed that their clinic had consultation rooms that catered for diabetic patients only. Three-quarters $(75.8 \%, n=113)$ of the respondents were of the opinion that all people should go for retinal screening, $73.8 \%(n=110)$ indicated that their facility had a retinal screening clinic and $71.8 \%(n=107)$ observed that retinal services were offered to all diabetics. The Chisquare test statistic of $83.25 \%$ was obtained when the frequency response patterns to the eight questions of this section were analysed. The $p$-value $(d f=28)$ associated with this result $(<0.0001)$ indicated that the significance level of $0.1 \%$ was reached. The conclusion is that the study participants did not respond to all questions in the same way.

TABLE 2: Study participants' knowledge about diabetic retinopathy. Subset of questions that describe/probe retinopathy awareness

. Diabetic retinopathy leads to permanent eye damage.

2.2: Diabetic retinopathy is well known in the community.

2.3: Diabetic retinopathy has been known to be common in people with DM for a long time.

2.4: Diabetic diseases result in diabetic retinopathy.

2.5: If my eyes itch a lot, does this mean that I have diabetic retinopathy?

2.6: Awareness regarding diabetic retinopathy helps people seek timeous assistance.

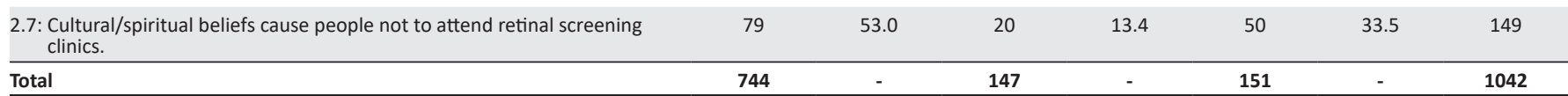

DM, diabetes mellitus.

TABLE 3: Factors related to diabetic retinal screening.

\begin{tabular}{|c|c|c|c|c|c|c|c|}
\hline \multirow{3}{*}{$\begin{array}{l}\text { Subset of questions on eye-screening procedures to prevent diabetic } \\
\text { retinopathy }\end{array}$} & \multicolumn{7}{|c|}{ Agreement levels } \\
\hline & \multicolumn{2}{|c|}{ Agree } & \multicolumn{2}{|c|}{ Undecided } & \multicolumn{2}{|c|}{ Disagree } & \multirow[t]{2}{*}{ Total } \\
\hline & $n$ & $\%$ & $n$ & $\%$ & $n$ & $\%$ & \\
\hline 3.1: People in our community understand the importance of screening. & 94 & 63.1 & 32 & 21.5 & 23 & 15.4 & 149 \\
\hline 3.2: Each health facility in this community has a diabetic clinic. & 128 & 85.9 & 14 & 9.4 & 7 & 4.7 & 149 \\
\hline $\begin{array}{l}\text { 3.3: At the clinic, there are consultation rooms that offer diabetic health } \\
\text { services only. }\end{array}$ & 127 & 85.2 & 14 & 9.4 & 8 & 5.4 & 149 \\
\hline 3.4: Our clinic has a retinal screening clinic. & 110 & 73.8 & 30 & 20.1 & 9 & 6.0 & 149 \\
\hline 3.5: Retinal screening services are offered to all diabetic patients. & 107 & 71.8 & 29 & 19.4 & 13 & 8.7 & 149 \\
\hline 3.6: Attending retinal screening sessions may delay the need for spectacles. & 90 & 60.4 & 25 & 16.8 & 34 & 22.8 & 149 \\
\hline 3.7: All people should go for retinal screening. & 113 & 75.8 & 22 & 14.8 & 14 & 9.4 & 149 \\
\hline 3.8: Diabetic staff make referrals for retinal screening when consulting. & 114 & 76.5 & 24 & 16.1 & 11 & 7.4 & 149 \\
\hline Total & 883 & - & 190 & - & 119 & - & 1192 \\
\hline
\end{tabular}


TABLE 4: Awareness of the risks associated with diabetes mellitus and diabetic retinopathy.

\begin{tabular}{|c|c|c|c|c|c|c|c|}
\hline \multirow{3}{*}{$\begin{array}{l}\text { Subset of questions that describe/probe diabetes } \\
\text { mellitus/retinopathy risk awareness }\end{array}$} & \multicolumn{7}{|c|}{ Agreement levels } \\
\hline & \multicolumn{2}{|c|}{ Agree } & \multicolumn{2}{|c|}{ Undecided } & \multicolumn{2}{|c|}{ Disagree } & \multirow[t]{2}{*}{ Total } \\
\hline & $n$ & $\%$ & $n$ & $\%$ & $n$ & $\%$ & \\
\hline 4.1: Most diabetic patients go for retinal screening. & 128 & 85.9 & 14 & 9.4 & 7 & 4.7 & 149 \\
\hline 4.2: Eating a healthy diet contributes to quality of life. & 143 & 96.0 & 3 & 2.0 & 3 & 2.0 & 149 \\
\hline 4.3: Doing regular exercises will keep me healthy. & 137 & 91.9 & 7 & 4.7 & 5 & 3.3 & 149 \\
\hline 4.4: I keep doctors' appointments for my eyes. & 124 & 83.2 & 11 & 7.4 & 14 & 9.4 & 149 \\
\hline 4.5: Smoking can elevate blood sugar levels. & 116 & 77.8 & 23 & 15.4 & 10 & 6.7 & 149 \\
\hline 4.6: Drinking alcohol can elevate blood sugar levels. & 120 & 80.5 & 21 & 14.1 & 8 & 5.4 & 149 \\
\hline 4.7: Overweight increases the risk of developing DM. & 129 & 86.6 & 12 & 8.0 & 8 & 5.4 & 149 \\
\hline Total & 897 & - & 91 & - & 55 & - & 1043 \\
\hline
\end{tabular}

DM, diabetes mellitus.

The final part of the questionnaire dealt with the participants' awareness of the risk factors associated with DM and DR. The results are summarised in Table 4 .

As shown in Table 4, most respondents were of the opinion that diabetic patients do go for retinal screening $(85.9 \%, n=$ 128). With respect to diet and eating habits, most respondents $(95.9 \%, n=143)$ agreed that eating a healthy diet contributes to the quality of life. Importantly, almost all $(91.9 \%, n=137)$ of the respondents agreed that doing regular exercise also contributes to quality of life. With respect to keeping their doctors' appointments for their eyes, 83.2\% $(n=124)$ of the respondents agreed with the statement, implying that most patients take their health seriously and honour their appointments. This may also be because of the high numbers of backlogged patients making it difficult to get a new appointment at the clinic. Regarding the consumption of alcohol and smoking, $80.5 \%(n=120)$ and $77.9 \%(n=116)$ of the respondents, respectively, agreed with the statement that these habits could elevate sugar levels. In addition, the majority of the respondents $(86.6 \%, n=129)$ agreed that being overweight increases the risk of developing DM. Items 4.2, 4.3, 4.6 and 4.7 in Table 4 address the major risks associated with DM and DR. The high level of agreement with the risk statement (ranging from $80.5 \%$ to $95.9 \%$, with a mean of $88.8 \%$ ) gives credence to the idea that respondents are generally aware of the risks associated with DM and DR.

\section{Discussion}

Data were collected from 149 female diabetics, of whom just over half $(54.3 \%, n=81)$ were between 51 and 60 years of age. In a similiar study, ${ }^{18}$ it was reported that the most important demographic change in the prevalence of DM across the world appears to be the increase in the proportion of older people. More than half of the respondents $(56.5 \%, n=84$ ) were single, divorced or widowed. This high number of unmarried women may be prone to defaulting their diabetes medication in order to provide for their families, because of the lack of support from a partner. IsiXhosa is the predominant language of the residents ${ }^{16}$ and $91.3 \%(n=136)$ of the respondents belonged to this language group. A low percentage of respondents were formally educated.

Only $21.5 \%(n=32)$ had completed Grade 11 or 12 , whilst a low percentage $(24.1 \%, n=36)$, had had some form of tertiary education. The importance of education was emphasised in a similar study conducted in Malaysia, which assessed the awareness of eye complications and the prevalence of DR. ${ }^{12}$ The present study found that $38.8 \%(n=57)$ of the participants were unemployed, with only $23.1 \%(n=34)$ reported being employed or self-employed. This was expected because of the lower levels of formal education achieved.

Over half of the respondents $(58 \%, n=80)$ reported a family history of DM, although $42 \%(n=58)$ reported no known family history of DM. Type 2 diabetes tends to occur even more frequently where there is a history of diabetes in the family. Studies of families ${ }^{19}$ have revealed that first-degree relatives or individuals with Type 2 diabetes are three times more likely to develop the disease than individuals without a positive family history.

Just over $70 \%(n=107)$ of the respondents agreed that DR is more common in people who have had DM for a long time. As the majority of the respondents had known their diabetic status for over 8 years, these were the respondents who had the most knowledge and awareness about DM and DR. Only $15 \%(n=22)$ of the respondents reported wearing spectacles for reading and near work purposes only whilst $39.7 \%(n=58)$ wore spectacles full time and had visual errors that needed spectacles to see at both near and far distance. In the diabetic club they encourage patients to have their retinal screening first; spectacles follow if the retinal photos show no abnormalities in the retina. A significant number of respondents $(45.2 \%, n=66)$ reported not wearing spectacles at all. These respondents were possibly waiting for appointments for spectacles, had never tested their eyes before or were not aware that they were able to receive an eye test and spectacles at this facility. Altogether $85 \%(n=127)$ of the respondents reported being satisfied or very satisfied with the information provided at the diabetic clinic with only $4.7 \%$ $(n=7)$ being dissatisfied with this information. This showed that the club was knowledgeable about diabetic eye care and they explained the information well to their club patients.

Most respondents displayed informed knowledge of general health and diabetes. Approximately $80 \%(n=119)$ agreed that the diabetes is related to high sugar intake; however, this statement usually applies to Type 2 diabetes if there is weight 
gain rather than related to Type 1 diabetes. Over half of the study participants $(n=80)$ agreed that DM is more common in adults than in children. Research published in $2006^{18}$ found that the prevalence of DM among all age groups worldwide was estimated to be $2.8 \%$ in 2000 and was expected to increase to $4.4 \%$ by 2030 . The most important demographic change to DM prevalence internationally appears to be the increase in the proportion of people older than 65 years of age. However, Type 2 is becoming prevalent amongst children, particularly in obese children. It is essentially a lifestyle disease that may be managed, to some extent, by exercise and diet. Overall, respondents demonstrated a fairly good general knowledge of DM, with almost $80 \%$ agreeing that DM can lead to blindness, although only about a half $(n=73)$ agreed that DM is common in obese people. The prevalence of DM is increasing owing to growing numbers in the older population, greater urbanisation and the increasing levels of obesity and physical inactivity through poor lifestyle changes. The $\mathrm{WHO}^{18}$ projected that the diabetes pandemic will continue to rise if levels of obesity remain constant. Above $50 \%$ of the respondents $(n=80)$ in the present study agreed that diabetes was more common in adults than in children. This ties in with the findings by Lacsar et al..$^{20}$ that the prevalence of Type 2 diabetes in adolescents as well as in young adults is increasing dramatically in South Africa. The major predisposing risk factors include obesity, family history of diabetes and sedentary lifestyle.

The study participants demonstrated moderate knowledge levels regarding the effects of DM on their eyes, and almost three-quarters of respondents $(71.1 \%, n=106)$ agreed that poor eyesight owing to DM affects one's quality of life. This important aspect was highlighted in a 2007 study $^{5}$ that found that DR is the fifth leading cause of blindness worldwide. The present study also found a generally high level of awareness about DR $(71.1 \%, n=106)$ among respondents. Eighty per cent of the respondents $(n=119)$ agreed that DR leads to permanent eye damage, and that DM results in DR. This finding resonates with the findings of the National Eye Institute $^{21}$ that DR affects blood vessels in the retina and is the most common cause of vision loss among people with diabetes and the leading cause of vision impairment and blindness among working people. With the longer duration of DM, most diabetic patients are prone to developing DR, particularly if blood sugars are not controlled properly. Because about a quarter $(24.5 \%, n=36)$ of participants in the present study had DM for over 8 years, they were at considerable risk of developing DR.

Almost three-quarters $(n=117)$ of the respondents agreed that DM causes DR. This is an important issue that all patients with DM should be aware of. In a study conducted in $2003,{ }^{8}$ it was established that almost all Type 1 diabetics will develop DR between 10 and 20 years of the patient becoming diabetic, whilst patients with Type 2 diabetes are at risk of developing DR. The prevalence of DR has been reported to be $35 \%$ in patients who have had diabetes for 12 years. ${ }^{22}$ On the contrary, a later study ${ }^{23}$ indicated that all diabetic patients are at a risk of DR; however, those living with Type 1 diabetes have a higher chance of developing this condition. In line with the $\mathrm{HBM}^{14}$ the respondents, who had an awareness of DR, appeared to attend the diabetic clinic regularly once a month. They also had knowledge of the possibility of being referred for retinal screening to assess their retinas for any diabetic changes. What is important here is that an awareness of the availability of retinal screening and the knowledge to attend such screenings at regular intervals is key to managing DR before advanced or sight-threatening retinopathy develops.

Over half $(n=99)$ of the respondents agreed with the statement that if their eyes itch a lot then this was an indication of DR, which showed a lack of understanding of the symptoms of DR. This might explain why many diabetic patients will demand spectacles on retinal screening days or believe that diabetes causes itchy eyes and that a pair of glasses will alleviate the itching. As the early stages of DR are almost symptomless, it is advised for every diabetic patient to have a retinal screening annually to detect early changes. However, just over half of the respondents $(n=79)$ indicated that they do not attend retinal screening because of cultural and spiritual beliefs. This strongly suggests that culture and beliefs play a role in how some of the respondents manage their diabetes and may subsequently impact on how they care for their eyesight. However, over 33\% $(n=50)$ of respondents disagreed with this statement. The majority of study respondents $(85 \%, n=128)$ were aware that each facility in the community had a diabetic clinic and had specific consultation rooms that offered diabetic health services only. Also, respondents demonstrated a reasonable level of awarenes of diabetic retinal screening, and just under two-thirds $(63 \%, n=94)$ agreed that the importance of retinal screening is understood by people in the community. There were also the respondents who were satisfied with the DM information about their eyes provided by the diabetic clinic. The importance of retinal screening was emphasised by Cook ${ }^{11}$ who observed that although diabetic retinal screening is advised as a preventative measure for all diabetic patients, it appears that some patients are not well informed about its importance and do not know where and how to have the retinal screening done.

A further important finding in the present study was that over $60 \%(n=90)$ of the respondents felt that retinal screening may delay the process of acquiring new spectacles, whilst only about $23 \%(n=34)$ disagreed about this. This indicates a lack of knowledge about the importance of retinal screening. It is very important for every diabetic to have retinal screening and to wait for the retinal results before they can be referred to acquire spectacles. However, the study also found that more than three-quarters of respondents $(n=114)$ understood that diabetic staff make referrals for retinal screening during consultation. Although ideally this awareness should be higher, it does suggest that protocols are in place for referral and management of the diabetic patients attending the club. About three-quarters $(n=113)$ of the respondents indicated that all people should go for retinal screening. However, not 
all people, but certainly all diabetic people should have undergone retinal screening.

A study by the National Eye Institute ${ }^{21}$ suggested that all people with DM - both Type 1 and Type 2 - were at a risk of sight-threatening DR, so it is generally recommended that all diabetic patients should undergo a comprehensive dilated eye examination at least once a year.

Over $85 \%(n=128)$ of the respondents in the present study agreed that most of the diabetic patients attend retinal screening. This statement was contrary to what was observed on some retinal screening clinic days, where the actual presentation of patients was very low. When it came to normal refractive screening days, for the prescription of spectacles, these numbers were moderately higher. In general, the knowledge about DR is positively reported in these results. In a study by Van Eijk et al. ${ }^{12}$ in the United States of America, it was found that financial barriers may cause diabetic patients not to attend retinal screening more than refractive screening; however, the main barrier to compliance appeared to be patients being in denial about having DR. Other factors included embarrassment about poor glycaemic control and their fear of being referred to an ophthalmologist for treatment. However, the knowledge reported appears to be contrary to the observation noted in practice that diabetic patients will sometimes report to the eye clinic with DR when they are in the advanced stages of diabetes. The question that may be asked regarding this contradiction would be, what causes the patients not to return for their retinal results or follow-ups after their retinal screening?

Some study limitations need to be noted. Firstly, the data were collected from a single day clinic, so the results reflect the opinions of the sample from only one area. Secondly, the study involved a relatively small participant sample, so the results may not be generalisable to the community at large. Thirdly, the present study focussed on female patients attending the clinic and hence does not reflect any views across gender groups. Fourthly, most respondents were isiXhosa speaking, and they were required to answer the questionnaire only in English, which could have compromised some responses. Lastly, the study utilised convenience sampling; however, the purposive nature of the sampling, which included all diabetic patients attending the day clinic during the data collection period, does mitigate against possible selection bias from the non-random sampling process.

\section{Conclusion and recommendations}

With DM on the rise globally and fuelled by factors such as the increasing prevalence of obesity, the growth in urbanisation and unhealthy lifestyles, increased risk of preventable blindness because DR may be expected. This study examined the awareness levels of DR and retinal screening amongst female diabetic patients and concluded that although there was fair awareness about retinal screening and DR amongst such patients, significant knowledge gaps were identified amongst these patients with regard to DR knowledge and awareness. However, these shortcomings might be rectified together with increased knowledge about the prevention, treatment and management of DM.

The study has shown the need for important public health interventions in the surrounding day hospitals in Khayelitsha, Cape Town, to decrease the number of people at a risk of developing irreversible blindness. It is critically important that all diabetic patients attend diabetic clinics regularly, are informed and are timeously referred for retinal screening where necessary. However, because few facilities are fully operational and have the means to conduct intensive retinal screening, this issue needs to be addressed urgently as part of the overall health services provided for patients with a chronic disease. It is recommended that similar studies that involve both eye care workers and attending patients be conducted or replicated in other parts of the Western Cape and in South Africa as a whole. In addition, future studies could involve both male and female respondents to determine whether there are additional risk factors that may emerge from male respondents.

\section{Acknowledgements}

The authors would like to acknowledge Professor Lydia Monareng from the University of South Africa for her expert guidance and wealth of research knowledge, throughout this paper.

\section{Competing interests}

The authors declare that they have no financial or personal relationships that may have inappropriately influenced them when writing this article.

\section{Authors' contributions}

N.F.M. and P. C.-F. wrote this article, interpreted the data and read and approved the final version of the manuscript.

\section{Funding information}

The Master's and Doctoral Research Bursary Committee, from the University of South Africa, funded this study.

\section{Data availability}

Data sharing is not applicable to this article.

\section{Disclaimer}

The views and opinions expressed in this article are those of the authors and do not necessarily reflect the official policy or position of any affiliated agency of the authors. 


\section{References}

1. World Health Organization. Global report on diabetes [homepage on the Internet] 2016 [cited 2016 Feb 07]; p. 6. Available from: https://www.who.int/diabetes/ publications/grd-2016/en/

2. Harding JL, Pavkov ME, Magliano DJ, Shaw JE, Gregg E. Global trends in diabetes complications: A review of the current evidence. Diabetologia. 2019;62(3):3-16. https://doi.org/10.1007/s00125-018-4711-2

3. Clarke-Farr PC, Nel NM, Wilkinson A. An investigation into diabetic patients' knowledge of diabetes and its ocular complications in the Western Cape. S Afr Optom. 2006;65(4):134-143. https://doi.org/10.4102/aveh.v65i4.268

4. Phillips KC, Mashige KP, Clarke-Farr PC. Knowledge of diabetes mellitus in privately-funded diabetic patients attending a rural optometric practice in
Malmesbury, South Africa. S Afr Optom. 2012;71(2):1-8. https://doi.org/10.4102/ aveh.v71i2.71

5. Read $\mathrm{O}$, Cook C. Retinopathy in diabetic patients evaluated at primary health care in Cape Town. S Afr Med J. 2007;12(2):56-64. https://doi.org/10.1080/22201009. 2007.10872158

6. Rani PK, Raman R, Agarwal S, et al. Diabetic retinopathy screening model for rural population: Awareness and screening methodology. Rural Remote Health 2005;35(5):1-11. https://doi.org/10.22605/RRH350

7. Smith STT, Szetu J, Bourne RRA. Prevalence and severity of diabetic retinopathy, associated risk factors and vision loss in patients registered with type 2 diabetes in Luganville, Vanuata. Br J Ophthalmol. 2007;91(4):415-419. https://doi.org/10.1136/ bjo.2006.104174

8. Fong DS, Aiello L, Gardener TW, et al. Diabetic retinopathy. Diabetes Care. 2003;26(1):143-156. https://doi.org/10.2337/diacare.26.1.226

9. Mwangi MW, Githinji GG, Githinji F. Knowledge and awareness of diabetic retinopathy amongst diabetic patients in Kenyatta national hospital, Kenya. Int Humanit Soc Sci. 2011;21(1):140-146.

10. Vijan S, Hofer TP, Hayward R. Cost-utility analysis of screening intervals for diabetic retinopathy in patients with Type 2 diabetes mellitus. I Am Med Assoc. retinopathy in patients with Type 2 diabetes mellitus. J $A m$
2000;283(7):889-896. https://doi.org/10.1001/jama.283.7.889

11. Cook S. Diabetic retinopathy - The Ophthalmology Society of Southern Africa screening programme. S Afr Med J. 2013;103(7):449-451. https://doi.org/ 10.7196/SAMJ.7136
12. Tajunisah I, Wong PS, Tan LT, Rokiah P, Reddy S. Awareness of eye complications and prevalence of retinopathy in the first visit to the eye clinic among Type 2 diabetic patients. Int J Ophthalmol. 2011;4(5):519-524.

13. Nwosu S. Diabetic retinopathy: Management update. Niger Postgrad Med J. 2003;10(2):115-120.

14. Stanhope MLJ. Community and public health nursing. 5th ed. St Louis, MO: A Harcourt Health Sciences Company; 2000.

15. Tuner LW, Hunt SB, DiBrezzo R, Jones C. Design and implementation of an osteoporosis prevention program using the health belief model. Am J Heal Stud. 2004;19(2):115-121.

16. City of Cape Town. 2011Census_CT_Suburb_Khayelitsha_Profile [homepage on the Internet]. Cape Town; 2011 [cited 2013 Nov 04]. Available from: http://resource. capetown.gov.za/documentcentre/Documents/Mapsandstatistics/2011_Census_ CT_Suburb_Khayelitsha_Profile.pdf

17. Burns N, Grove S. The practice of nursing research: Appraisal, synthesis and generation of evidence. 6th ed. St Louis, MO: Saunders; 2009.

18. Rheeda P. Diabetes Type 2: The emerging epidemic. S Afr Fam. 2006;48(10):20. https://doi.org/10.1080/20786204.2006.10873480

19. World Health Organization. Diabetes mellitus control [homepage on the Internet]. 2014 [cited 2015 Jul 7]. Available from: http://www.afro.who.int/en/clusters-aprogrammes/dpc/noncommunicablediseases-manangementndm/programmecomponents/diabetesmellitus.html

20. Lascar N, Brown J, Pattison H, Barnett A, Bailey C, Bellary S. Type 2 diabetes in adolescents and young adults. Lancet Diabetes Endo. 2018;69(1):69-80. https:// doi.org/10.1016/S2213-8587(17)30186-9

21. National Eye Institute. Facts about diabetic retinopathy [homepage on the Internet]. 2013 [cited 2015 Mar 17]. Available from: https://www.nei.nih.gov/ health/diabetic/retinopathy

22. Tam J, Dhamddhere KP, Tiruveedhula P, et al. Disruption of the retinal parafoveal capillary network in Type 2 diabetes before the onset of diabetic retinopathy. Invest Ophthalmol Vis Sci. 2011;52(12):9257-9266. https://doi.org/10.1167/iovs.11-8481

23. Van Eijk KND, Blom JW, Gussekloo J, Polak BCP, Groeneveld Y. Diabetic retinopathy screening in patients with diabetic mellitus in Primary Health Care: Incentives and barriers to screening attendance. Diabetes Res Clin Pract. 2011;2012(96):10-16. https://doi.org/10.1016/j.diabres.2011.11.003 